\title{
A synthetic diamond diode in volumetric modulated arc therapy dosimetry
}

\author{
Margherita Zani, ${ }^{\text {a) }}$ Marta Bucciolini, Marta Casati, and Cinzia Talamonti \\ Dipartimento di Scienze biomediche, sperimentali e cliniche, Università degli Studi di Firenze - Azienza \\ Ospedaliero Universitaria Careggi, Largo Brambilla 3, I-50134 Firenze, Italy \\ Marco Marinelli, Giuseppe Prestopino, Alessia Tonnetti, and Gianluca Verona-Rinati \\ INFN-Dipartimento di Ingegneria Industriale, Università di Roma "Tor Vergata", Via del Politecnico 1, \\ I-00133 Roma, Italy
}

(Received 29 May 2013; revised 11 July 2013; accepted for publication 30 July 2013; published 16 August 2013)

Purpose: The aim of this work is to investigate the behavior of a single crystal diamond diode (SCDD) for volumetric modulated arc therapy (VMAT) dose verifications. This delivery technique is one of the most severe test of a dosimeter performance due to the modulation of the dose rate achieved by simultaneously changing the velocity of the gantry and the position of the collimator leaves. The performed measurements with VMAT photon beams can therefore contribute to an overall global validation of the device to be used in dose distribution verifications.

Methods: The SCDD response to 6 MVRX has been tested and compared with reference ionization chambers and treatment planning system (TPS) calculations in different experiments: (a) measurements of output factors for small field sizes (square fields of side ranging between $8 \mathrm{~mm}$ and $104 \mathrm{~mm}$ ) by SCDD and A1SL ionization chamber; (b) angular dependence evaluation of the entire experimental set-up by SCDD, A1SL, and Farmer ionization chambers; and (c) acquisition of dose profiles for a VMAT treatment of a pulmonary disease in latero-lateral and gantry-target directions by SCDD and A1SL ionization chamber.

Results: The output factors measured by SCDD favorably compare with the ones obtained by A1SL, whose response is affected by the lack of charged particle equilibrium and by averaging effect when small fields are involved. From the experiment on angular dependence, a good agreement is observed among the diamond diode, the ion chambers, and the TPS. In VMAT profiles, the absorbed doses measured by SCDD and A1SL compare well with the TPS calculated ones. An overall better agreement is observed in the case of the diamond dosimeter, which is also showing a better accuracy in terms of distance to agreement in the high gradient regions.

Conclusions: Synthetic diamond diodes, whose performance were previously studied for conformal and IMRT radiotherapy techniques, were found to be suitable detectors also for dosimetric measurements in volumetric arc therapy treatments. (C) 2013 American Association of Physicists in Medicine. [http://dx.doi.org/10.1118/1.4818256]

Key words: radiation dosimetry, semiconductor detectors, treatment verifications

\section{INTRODUCTION}

Nowadays, patients are often treated in radiotherapy by using uniform and/or nonuniform fields, composed of small segments and characterized by high dose gradients, variation in space and time of the dose rate and beam energy spectrum. The dosimetry of the small and/or nonstandard radiation fields used in these high conformal techniques represents a new challenge for the medical physicist community. ${ }^{1-3}$ In fact, under these conditions, measurements are performed when the charged particle equilibrium (CPE) requirement is not fulfilled, so that the presence of the detector can strongly perturb the charged particle fluence. The extent of the effects due to the lack of CPE depends on the detector physical and electron densities as well as on the detector geometry, besides being related to the beam energy spectrum and the spatial conformation of the radiation field. In order to minimize the perturbation on the radiation field due to the detector, it is essential for the materials constituting the device to be phantom equiva- lent as much as possible and that the dosimeter has very small dimensions. This last requirement allows to minimize the averaging effect in the presence of nonuniform radiation fields. ${ }^{4}$ Diamond detectors seem to be the best candidates for point dose measurements in this kind of highly conformal radiotherapy techniques. ${ }^{5-9}$

A PTW natural diamond based dosimeter has been previously used for IMRT applications,,${ }^{10,11}$ but its use is limited because of high costs and low availability of detector grade natural diamonds. The behavior of synthetic diamonds for IMRT dosimetry has been analyzed in a few papers. ${ }^{6-8,12-14}$ In particular, in the paper by Almaviva, ${ }^{6}$ very promising features of a synthetic single crystal diamond detector (SCDD) developed at Roma Tor vergata University are shown. These SCDDs have been tested as radiotherapy dosimeters with different beam qualities (photons from 60 Co to $10 \mathrm{MV}$ and electrons from $6 \mathrm{MeV}$ to $18 \mathrm{MeV}$ ). ${ }^{6,15-17}$

In the present work, the dosimetric properties of a SCDD were tested by studying point dose measurements during 
volumetric modulated arc therapy (VMAT) treatments. This delivery technique poses stringent requirements on the dosimetric performances of a detector due to the modulation of the dose rate achieved changing the velocity of the gantry and of the collimator leaves. The detector experiences variable beam energy distribution and fluence: during an irradiation session, it happens that it is positioned for a fraction of time under closed leaves and, for another time interval, under the open field or at the edge of the field while the beam orientation is also changing. Hence, all the requirements for a good detector in dosimetry, such as time stability, good linearity, no energy dependence, no dose rate dependence, high spatial resolution, no angular dependence, and fast response dynamics need to be satisfied in a stringent way. The measurements performed with VMAT photon beams concur to the validation of the device to be used in dose distribution verifications.

In order to experimentally verify the patient dose distribution calculated by the treatment planning system (TPS) before the delivery, the standard procedure is to use a solid water or PMMA phantom (QA phantom). The actual intensity map is exported to the phantom, where the dose distribution is recalculated. The comparison between calculations and experimental data can be performed either by point dose verifications or with two dimensional detectors. Generally the latter is the preferred solution, as it permits to obtain multiple data points in a single treatment delivery. Some commercial 2D detectors are available, constituted by a matrix of ion chambers (MATRIXX ${ }^{\mathrm{TM}}$ IBA, OCTAVIUS ${ }^{\mathrm{TM}}$ PTW), or of silicon diodes (MAPCHECK ${ }^{\mathrm{TM}}$ Sun Nuclear); their performances are discussed in several papers. ${ }^{18-22}$ Ion chambers, due to the low specific sensitivity, present the drawback of a low spatial resolution, while the silicon diodes, because of the high $\mathrm{Z}$ material, can suffer of an energy dependent response. In principle, a matrix of very small diamond dosimeters, with a millimetric pitch, could be the best solution. A matrix of diamond detectors is not on the market yet, but the work presented in this paper, where the performances of the point diamond dosimeter have been tested using a complex technique as VMAT, is a milestone for realizing such a device.

\section{MATERIALS AND METHODS}

\section{A. Radiation beam and treatment technique}

An Elekta Synergy Beam Modulator Linac was used for the beam delivery. The LINAC has a fixed jaw which determines the maximum field size $16 \mathrm{~cm} \times 21 \mathrm{~cm}$ and is equipped by an 80 -leaves multileaf collimator, having a $4 \mathrm{~mm}$ leaf width (at isocenter). The smallest symmetric field achievable is $0.8 \mathrm{~cm} \times 0.8 \mathrm{~cm}$. Among the three available photon energies $(6 \mathrm{MV}, 10 \mathrm{MV}, 18 \mathrm{MV})$, the $6 \mathrm{MVRX}$ beam quality was chosen for the measurements discussed in the paper.

As rotational techniques are involved, the detector response versus irradiation direction was analyzed. In this kind of study, two parameters have a major role: the angular dependence of the dosimeter and the attenuation introduced by the patient couch, i.e., an iBEAM evo (Medical Intelligence) in the adopted setup. The couch has a thickness of $5 \mathrm{~cm}$ and

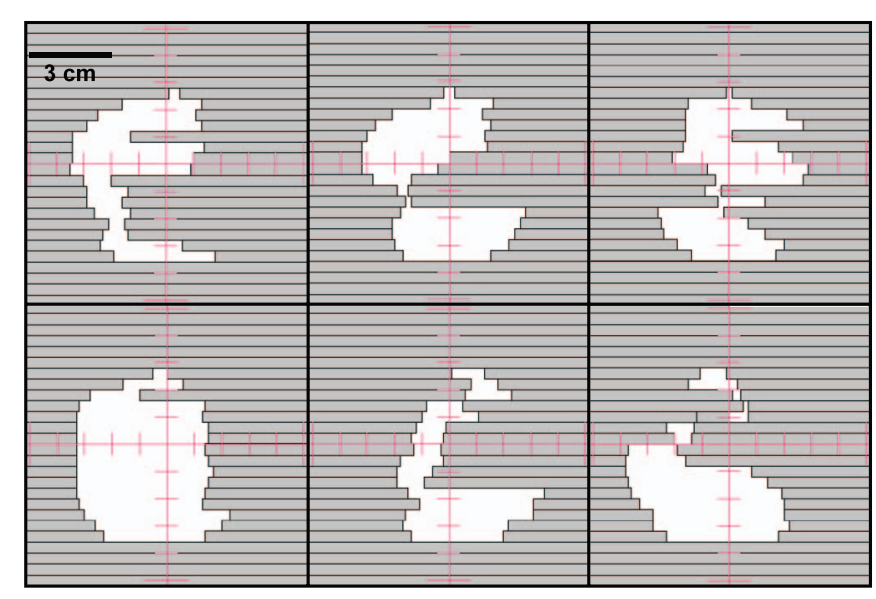

FIG. 1. Example of six different configurations (control points) of LINAC leaves during the considered VMAT treatment.

it is constituted by an external $2 \mathrm{~mm}$ thick carbon fiber filled with a low density foam core; a computed tomography (CT scan) of it was acquired by a Philips Brilliance Big Bore CT and used by the TPS in order to account for its attenuation $(2.5 \%)$.

A squared field $10.4 \mathrm{~cm} \times 10.4 \mathrm{~cm}$ was used for detector calibrations and for angular dependence study. Squared fields with side from $0.8 \mathrm{~cm}$ to $10.4 \mathrm{~cm}$ were used for the output factor measurements. A VMAT treatment planned with Philips Pinnacle ${ }^{3}$ Smart Arc tool and actually delivered to a patient affected by a pulmonary disease, was chosen to perform the validation of the SCDD. The treatment consists of a two arcs arrangement (clockwise $179^{\circ} \rightarrow 180^{\circ}$ and counterclockwise $180^{\circ} \rightarrow 179^{\circ}$ ), each one with 91 control points. $60 \mathrm{~Gy}(20 \mathrm{~Gy} / \mathrm{fr})$ were prescribed at the isocenter. As an example, the configurations of the leaves in six control points are shown in Fig. 1. The entire treatment fraction was delivered in about 7 min. The Pinnacle ${ }^{3}$ VMAT and LINAC settings adopted for the dosimetric characterization performed in this work are summarized in Table I.

\section{B. Detectors and phantoms}

The tested diamond detector consisted in a single crystal diamond Schottky diode grown by microwave chemical vapor deposition (CVD) at the University of Rome Tor Vergata. ${ }^{17}$ The device was incapsulated in the same PTW Freiburg housing used for the unshielded Silicon diode (PTW Diode E model 60017). Diamond sensitive volume is a cylinder $2.2 \mathrm{~mm}$ in diameter and about $1 \mu \mathrm{m}$ thick, placed $0.8 \mathrm{~mm}$ from the detector tip. A sketch of the SCDD is shown in Fig. 2(a). An Extradin A1SL ion chamber was used for comparison [Fig. 2(b)].

Absolute dose calibration was performed with a Farmer ionization chamber (PTW 30011), calibrated in terms of dose to water in a reference laboratory, coupled to an IBA Dose 1 electrometer, according to the IAEA TRS-398 code of practice. ${ }^{23}$ A1SL and SCDD were calibrated by intercomparison with the Farmer chamber in solid water, using a $10.4 \mathrm{~cm} \times 10.4 \mathrm{~cm}$ field, at the isocenter position. Two 
TABLE I. Pinnacle ${ }^{3}$ VMAT and LINAC settings relative to the dosimetric measurements performed in this work.

\begin{tabular}{lcc}
\hline \hline & Pinnacle $^{3}$ VMAT machine settings & LINAC settings \\
\hline Gantry speed & $5.5 \mathrm{deg} / \mathrm{s}$ & $6 \mathrm{deg} / \mathrm{s}$ \\
MLC leaf speed & $2.25 \mathrm{~cm} / \mathrm{s}$ & $2.5 \mathrm{~cm} / \mathrm{s}$ \\
Minimum dynamic leaf gap & $0.2 \mathrm{~cm}$ & $0.14 \mathrm{~cm}$ \\
Dose rate behavior (nominal values) & Binned: $50,100,200,400 \mathrm{MU} / \mathrm{min}$ & $50,100,200,400 \mathrm{MU} / \mathrm{min}$ \\
Maximum gantry MU delivery & $20 \mathrm{MU} / \mathrm{deg}$ & \\
Minimum gantry MU delivery & $0.1 \mathrm{MU} / \mathrm{deg}$ & \\
Minimum MLC leaf MU delivery & $0.3 \mathrm{MU} / \mathrm{cm}$ & \\
\hline \hline
\end{tabular}

different phantoms were used in the present study. A cylindrical PMMA phantom with a diameter of $15 \mathrm{~cm}$, having a circular hole in the center, along the whole longitudinal dimension; the phantom is equipped with an additional cylindrical PMMA plug to permit the detector positioning and the axial sliding in gantry-target (GT) direction ( $y$-axis), avoiding any air gap formation. The commercial IBA I'mRT phantom, was also used, made of a water equivalent material (RW3). In this case, the detector can be moved in different positions along latero-lateral (LL) direction ( $x$-axis).

Both the SCDD and the ion chamber were positioned with their axis parallel to the $y$-axis.

Both phantoms were CT scanned (Phillips Brilliance Big Bore CT) and images sent to the Pinnacle ${ }^{3}$ TPS. Figure 3 reports a scheme of the two phantoms.

The I'mRT phantom was used for two set of measurements: (a) Output factors for small fields with A1SL ionization chamber and SCDD and (b) VMAT treatment profile in LL direction. The cylindrical PMMA phantom was employed for two set of measurements as well: (c) Angular dependence of the entire experimental set-up and (d) VMAT treatment profile in GT direction. For each series of measurements, Pinnacle ${ }^{3}$ TPS was used for calculations.

\section{C. Comparison between measurements and calculations}

The dose distribution inside the phantom was calculated with a grid matrix of $1 \mathrm{~mm} \times 1 \mathrm{~mm} \times 1 \mathrm{~mm}$ resolution. Dose (a)

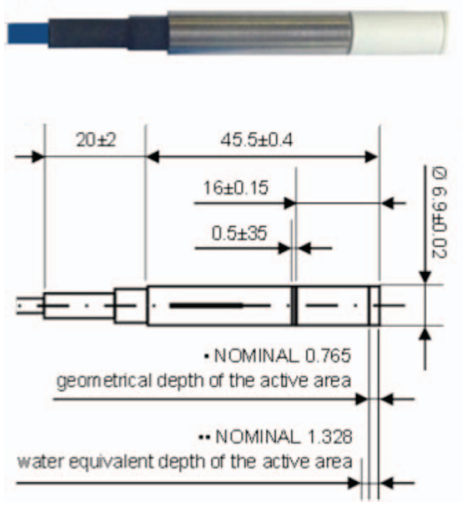

(b)

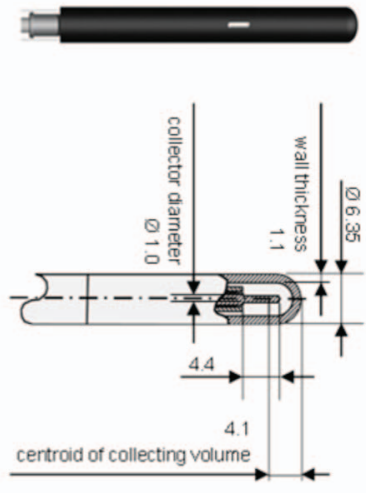

FIG. 2. Sketches of (a) SCDD and (b) A1SL ion chamber. profiles in the LL and GT directions passing through the isocenter were exported from the TPS. Profiles were acquired both with the A1SL ion chamber and with the SCDD, and for each device the VMAT treatment delivery was repeated with the detector placed in different positions. The agreement between measured and calculated doses was analyzed in terms of either absolute dose comparison and distance-to-agreement (DTA) ${ }^{24}$ defined as the distance between a measured dose point and the nearest calculated point with the same dose. In low dose gradient regions, the agreement was evaluated simply by the dose difference (DD), while the DTA represents the most meaningful quantity in regions where the spatial dose gradient is high. In our analysis, the dose gradient was evaluated by taking into account the six nearest neighbors of each point in the 3D grid TPS matrix. A threshold of $3 \% / \mathrm{mm}$ was assumed in the present study in order to discriminate between low and high dose gradient regions. ${ }^{25}$

\section{RESULTS AND DISCUSSION}

\section{A. Output factors}

Output factors for squared fields with side ranging from 0.8 to $10.4 \mathrm{~cm}$ were measured by A1SL ion chamber and SCDD. In Fig. 4, the response of the two dosimeters normalized to their response under a $10.4 \times 10.4 \mathrm{~cm}^{2}$ radiation field is shown. The values for the two detectors are in good agreement for fields wider than $1.6 \times 1.6 \mathrm{~cm}^{2}$ (maximum difference of $0.9 \%)$. For the smallest field $\left(0.8 \times 0.8 \mathrm{~cm}^{2}\right)$ the SCDD output factor is $15 \%$ higher with respect to the ion chamber. This difference can be ascribed to the difference in size of the two dosimeters (see the scheme of the two dosimeters reported in Fig. 2). Indeed, due to the relatively large volume of the A1SL chamber, both the lack of CPE and volume averaging become meaningful for small radiation fields. Such effects are less relevant in the case of SCDD. The smaller dimension of the SCDD and its higher density result in a better spatial resolution, a reduced averaging effect and a minimized loss of CPE. ${ }^{2}$

\section{B. Angular dependence}

A set of measurements at different gantry angles in the interval $0^{\circ} \div 360^{\circ}$ with $45^{\circ}$ steps was performed. The field used was $10.4 \times 10.4 \mathrm{~cm}^{2}$ and an intercomparison between the diamond dosimeter, A1SL, and Farmer ion chambers was 
(a)

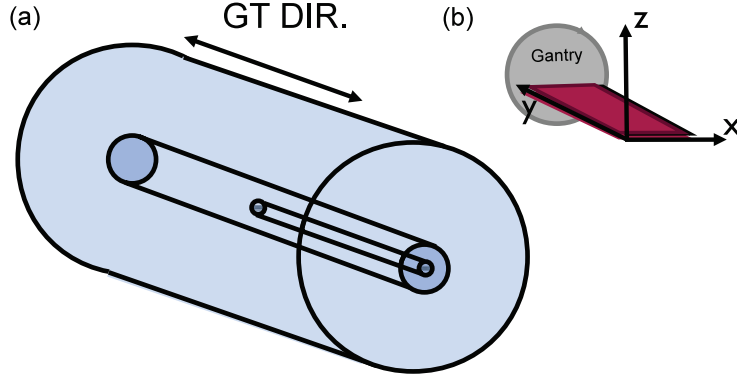

(c)

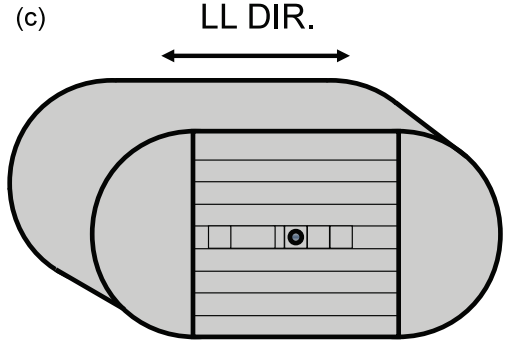

FIG. 3. Sketches of the cylindric PMMA phantom (a), the coordinate system and patient bed (b), and the IBA I'mRT phantom (c).

carried out, positioning all the detectors inside the cylindrical PMMA phantom, with their axis parallel to the $y$ direction (see Fig. 3). The dose measured by the three dosimeters was normalized with respect to the one acquired for the $0^{\circ}$ gantry angle and was also compared with the TPS calculation. The obtained results are shown in Fig. 5. Considering the Farmer chamber as a reference, it is observed that TPS data are in good agreement, since the maximum deviation is about $0.5 \%$. This finding confirms that the couch attenuation is properly modeled. The other two detectors both show a mean difference of $0.2 \%$ with respect to the reference, with a maximum deviation of $0.8 \%$ for A1SL and $0.5 \%$ for SCDD. This result demonstrates that, in this device orientation, the angular dependence of the diamond detector is negligible.

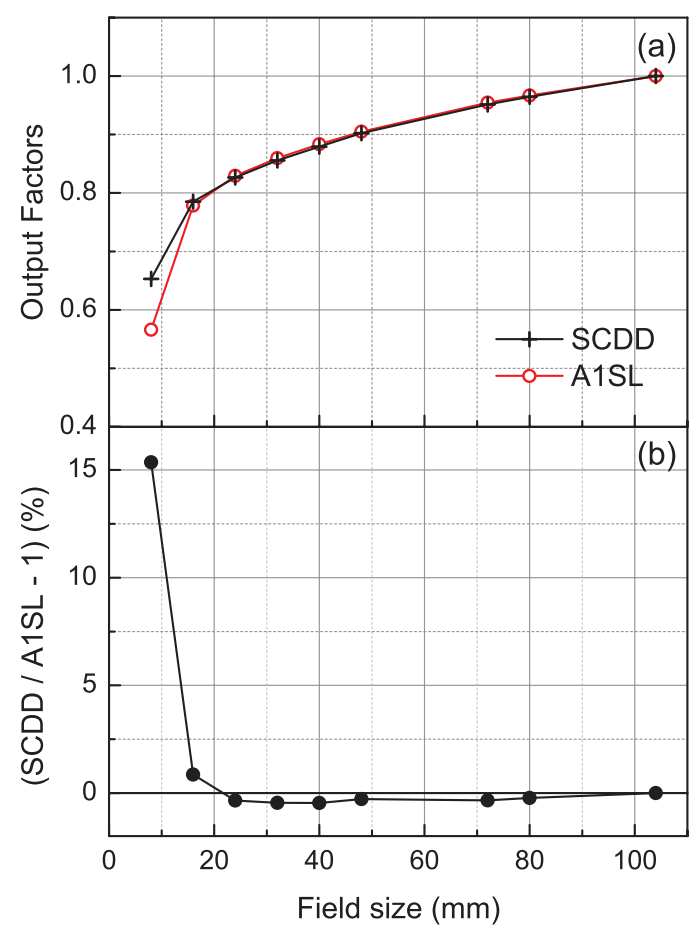

FIG. 4. (a) Output factors measured with A1SL (circles) and SCDD (crosses) in the range $0.8 \div 10.4 \mathrm{~cm}$. (b) Relative difference of the two detectors response in the same field size range.

\section{C. VMAT profiles}

\section{C.1. Gantry-target profile}

The results of the comparison between TPS calculations and measured values for the GT profile are summarized in Table II and shown in Fig. 6. Three different regions are considered: (1) central region, characterized by a low dose gradient, (2) penumbra region, and (3) tail of the distribution.

In the central region, an overall agreement is observed between SCDD and A1SL, but the diamond detector data are always closer to the TPS values. The SCDD dose difference is everywhere less than $2 \%$, with the exception of the point at $y=-2 \mathrm{~cm}$, where DD is $3.0 \%$. However, it should be noted that this value is confirmed by the A1SL measurement, being $0.8 \%$ the deviation between the two detectors. A similar behavior is observed at $y=-3 \mathrm{~cm}$, where both detectors measure a dose lower of about $2 \%$ respect to the calculation, but they are in good agreement.

Also in the penumbra region, the SCDD is globally in a better agreement to the TPS data, with an estimated DTA $\leq 0.6 \mathrm{~mm}$. A higher DTA of $2.2 \mathrm{~mm}$ is obtained at $y=-4 \mathrm{~cm}$ only, where a similar value is obtained by the ion chamber as well.

In the points on the tail of the distribution the dose difference is quite elevated (up to about $20 \%$ ). In this region, the dose gradient is low and the absorbed dose is about $5 \%$ of

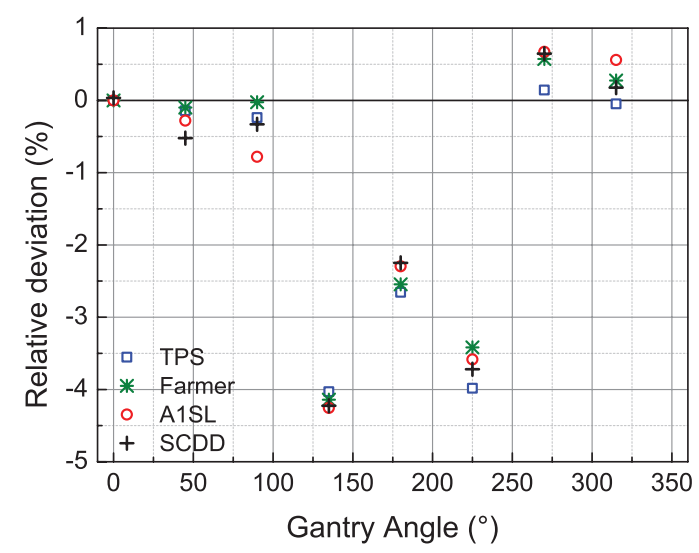

FIG. 5. Angular dependence of SCDD, A1SL, and Farmer ion chamber compared with the TPS calculation. Data are normalized at the $0^{\circ}$ gantry value. 
TABLE II. Measured points in GT profiles ( $y$ positions), calculated dose, estimated relative gradient, DD, and DTA for A1SL and SCDD.

\begin{tabular}{|c|c|c|c|c|c|c|c|}
\hline & \multirow[b]{2}{*}{$\begin{array}{c}Y \\
(\mathrm{~cm})\end{array}$} & \multirow[b]{2}{*}{$\begin{array}{l}\text { TPS dose } \\
\text { (cGy) }\end{array}$} & \multirow[b]{2}{*}{$\begin{array}{l}\text { Gradient } \\
(\% / \mathrm{mm})\end{array}$} & \multicolumn{2}{|c|}{ A1SL } & \multicolumn{2}{|c|}{ SCDD } \\
\hline & & & & $\begin{array}{l}\mathrm{DD} \\
(\%)\end{array}$ & $\begin{array}{l}\text { DTA } \\
(\mathrm{mm})\end{array}$ & $\begin{array}{l}\mathrm{DD} \\
(\%)\end{array}$ & $\begin{array}{l}\text { DTA } \\
(\mathrm{mm})\end{array}$ \\
\hline \multirow[t]{2}{*}{ Tail } & -6.0 & 116 & 2.2 & $-15.4 \%$ & $\ldots$ & $-21.3 \%$ & $\ldots$ \\
\hline & -5.0 & 142 & 2.6 & $-0.9 \%$ & $\ldots$ & $-0.2 \%$ & $\ldots$ \\
\hline \multirow[t]{3}{*}{ Penumbra } & -4.0 & 274 & 19.8 & $\ldots$ & 2.8 & $\ldots$ & 2.2 \\
\hline & -3.7 & 748 & 72.5 & $\ldots$ & 1.3 & $\ldots$ & 0.6 \\
\hline & -3.5 & 2034 & 27.4 & $\ldots$ & 0.1 & $\ldots$ & 0.6 \\
\hline \multirow[t]{5}{*}{ Low gradient } & -3.0 & 2582 & 2.6 & $-2.4 \%$ & $\ldots$ & $-1.9 \%$ & $\ldots$ \\
\hline & -2.0 & 2292 & 1.4 & $3.8 \%$ & $\ldots$ & $3.0 \%$ & $\ldots$ \\
\hline & -1.0 & 2368 & 0.5 & $2.2 \%$ & $\ldots$ & $0.2 \%$ & $\ldots$ \\
\hline & 0.0 & 2334 & 0.6 & $3.7 \%$ & $\ldots$ & $1.1 \%$ & $\ldots$ \\
\hline & 1.0 & 2301 & 0.9 & $3.5 \%$ & $\ldots$ & $1.3 \%$ & $\ldots$ \\
\hline \multirow[t]{3}{*}{ Penumbra } & 2.0 & 1629 & 11.0 & $\ldots$ & 0.2 & $\ldots$ & 0.5 \\
\hline & 2.5 & 770 & 27.8 & $\ldots$ & 0.6 & $\ldots$ & 0.4 \\
\hline & 3.0 & 223 & 19.3 & $\ldots$ & 1.0 & $\ldots$ & 0.1 \\
\hline \multirow[t]{3}{*}{ Tail } & 4.0 & 127 & 3.0 & $-7.8 \%$ & $\ldots$ & $-10.9 \%$ & $\ldots$ \\
\hline & 5.0 & 101 & 2.2 & $-13.3 \%$ & $\ldots$ & $-18.0 \%$ & $\ldots$ \\
\hline & 6.0 & 83 & 1.8 & $-16.6 \%$ & $\ldots$ & $-19.7 \%$ & $\ldots$ \\
\hline
\end{tabular}

the isocenter value. Such a dose is only due to the diffused radiation contribution and to the transmission under closed leaves. A similar extent of disagreement is observed between both detectors and TPS, thus indicating that in our TPS the dose under closed leaves is overestimated. The occurrence of this behavior was already observed by the authors during the commissioning of the Pinnacle ${ }^{3}$ system for VMAT treatments and measured both with ion chambers, radiochromic films, and 2D matrices of diode detectors, but it was accepted since it is protective for the patient and it does not introduce any noticeable effect in the irradiated volume.

\section{C.2. Latero-lateral profile}

The results concerning the absorbed doses measured in the LL profile ( $x$ direction) are presented in Table III and reported

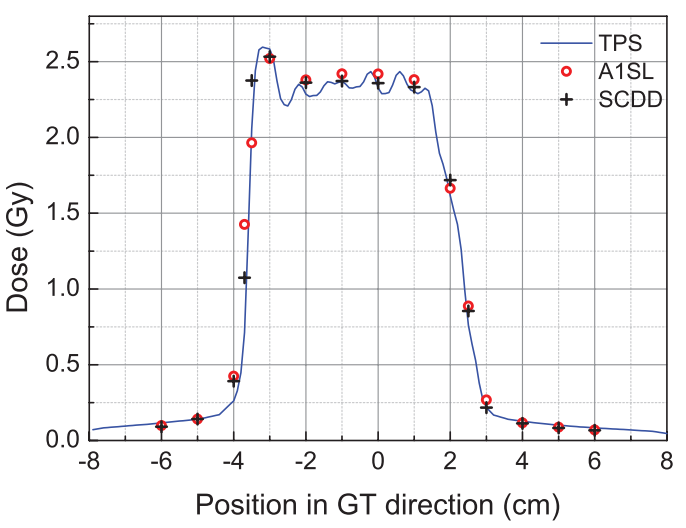

FIG. 6. Gantry-target isocenter profile measured with A1SL (circles), SCDD (crosses), and TPS calculated (blue line) inside the cylindrical PMMA phantom. in Fig. 7. The same criteria used for the analysis of the GT profile data were adopted. In this case, a tail region is not observed, being the dose at the extremities of the measured profile about $25 \%$ of the isocenter dose.

In the low gradient regions, it is possible to observe a deviation of the SCDD from the TPS up to about 3\%. However, it should be noted that, as already observed in the GT profile data, the agreement between the two detectors is always very good, with a maximum discrepancy of $1.3 \%$, measured at the isocenter. In the penumbra regions, the estimated DTA is always low $(\leq 1.1 \mathrm{~mm})$ for both the detectors.

It is worth to point out that, during a VMAT treatment, both the dose rate and the local dose per pulse are continuously varying as well. The good agreement of the diamond diode in such irradiation conditions, with both the reference dosimeter and the TPS, indicates that the device response is expected to be independent of the PRF and the dose per pulse variation.

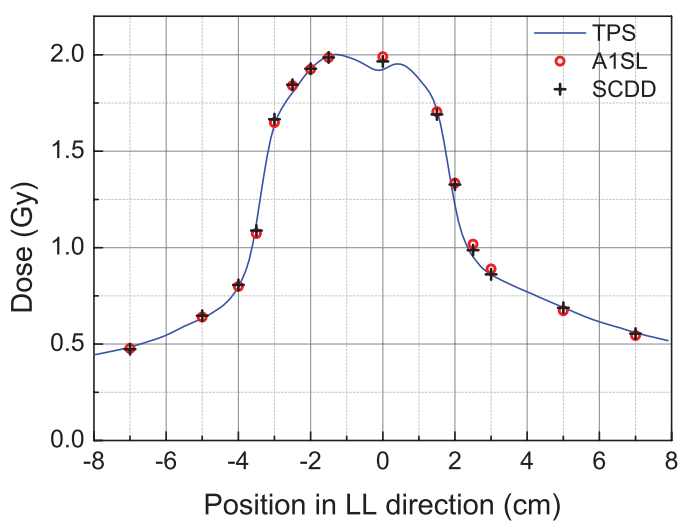

FIG. 7. Latero-lateral isocenter profile measured with A1SL (circles), SCDD (crosses), and TPS calculated (blue line) inside the IBA I'mRT phantom. 
TABLE III. Measured points in LL profile ( $x$ positions), calculated dose, estimated relative gradient, DD, and DTA for A1SL and SCDD.

\begin{tabular}{|c|c|c|c|c|c|c|c|}
\hline & \multirow[b]{2}{*}{$\begin{array}{c}X \\
(\mathrm{~cm})\end{array}$} & \multirow[b]{2}{*}{$\begin{array}{l}\text { TPS dose } \\
\text { (cGy) }\end{array}$} & \multirow[b]{2}{*}{$\begin{array}{l}\text { Gradient } \\
(\% / \mathrm{mm})\end{array}$} & \multicolumn{2}{|c|}{ A1SL } & \multicolumn{2}{|c|}{ SCDD } \\
\hline & & & & $\begin{array}{l}\text { DD } \\
(\%)\end{array}$ & $\begin{array}{l}\text { DTA } \\
(\mathrm{mm})\end{array}$ & $\begin{array}{l}\text { DD } \\
(\%)\end{array}$ & $\begin{array}{l}\text { DTA } \\
(\mathrm{mm})\end{array}$ \\
\hline \multirow[t]{2}{*}{ Low gradient } & -7.0 & 487 & 2.6 & $-2.0 \%$ & $\ldots$ & $-2.8 \%$ & $\ldots$ \\
\hline & -5.0 & 633 & 1.5 & $1.0 \%$ & $\ldots$ & $2.1 \%$ & $\ldots$ \\
\hline \multirow[t]{3}{*}{ Penumbra } & -4.0 & 800 & 4.4 & $\ldots$ & 0.0 & $\ldots$ & 0.2 \\
\hline & -3.5 & 1120 & 10.3 & $\ldots$ & 0.4 & $\ldots$ & 0.3 \\
\hline & -3.0 & 1630 & 4.3 & $\ldots$ & 0.3 & $\ldots$ & 0.5 \\
\hline \multirow[t]{4}{*}{ Low gradient } & -2.5 & 1800 & 2.0 & $2.2 \%$ & $\ldots$ & $2.5 \%$ & $\ldots$ \\
\hline & -2.0 & 1923 & 1.5 & $0.1 \%$ & $\ldots$ & $0.2 \%$ & $\ldots$ \\
\hline & -1.5 & 1991 & 0.7 & $-0.2 \%$ & $\ldots$ & $-0.2 \%$ & $\ldots$ \\
\hline & 0.0 & 1931 & 1.5 & $3.1 \%$ & $\ldots$ & $1.8 \%$ & $\ldots$ \\
\hline \multirow[t]{6}{*}{ Penumbra } & 1.5 & 1691 & 5.5 & $\ldots$ & 0.1 & $\ldots$ & 0.0 \\
\hline & 2.0 & 1185 & 11.4 & $\ldots$ & 1.1 & $\ldots$ & 1.1 \\
\hline & 2.5 & 921 & 10.2 & $\ldots$ & 1.0 & $\ldots$ & 0.7 \\
\hline & 3.0 & 830 & 10.6 & $\ldots$ & 0.7 & $\ldots$ & 0.4 \\
\hline & 5.0 & 682 & 5.0 & $\ldots$ & 0.3 & $\ldots$ & 0.2 \\
\hline & 7.0 & 555 & 3.6 & $\ldots$ & 0.6 & $\ldots$ & 0.0 \\
\hline
\end{tabular}

\section{CONCLUSIONS}

Aim of this work was to verify that the studied synthetic diamond diode is a suitable detector for verifying volumetric modulated arc therapy treatment. The obtained results confirm an overall good agreement between the SCDD dosimeter with both TPS and the reference ion chambers. In particular:

- The SCDD output factors favorably compare with A1SL values for which the lack of CPE and the averaging effect causes an underestimation of absorbed dose in small fields.

- Angular dependence measurements evidenced a good agreement between the diamond diode, the ion chambers, and the TPS.

- The VMAT profiles by SCDD and A1SL are found to be comparable in terms of dose difference and distance to agreement. However, an overall better agreement with respect to the TPS was observed for the diamond dosimeter, which is also showing a better accuracy in the high gradient regions with respect to the A1SL chamber.

The results obtained in the present study confirm the suitability of synthetic diamond diodes for point dose VMAT radiotherapy dosimetry. The development of 2D or 3D arrays of such diamond diodes would allow high accuracy in treatment plan verifications.

\section{ACKNOWLEDGMENTS}

The authors wish to thank Fondazione Roma for financial support and PTW Freiburg for providing the diamond housing and for helpful discussion.
a)Electronic mail: margherita.zani@unifi.it

${ }^{1}$ R. Alfonso, P. Andreo, R. Capote, M. S. Huq, W. Kilby, P. Kjall, T. R. Mackie, H. Palmans, K. Rosser, J. Seuntjens, W. Ullrich, and S. Vatnisky, "A new formalism for reference dosimetry of small and nonstandard fields," Med. Phys. 35, 5179-5186 (2008).

${ }^{2}$ I. J. Das, A. G. X. Ding, and A. Ahnesjö, "Small fields: Nonequilibrium radiation dosimetry," Med. Phys. 35, 206-215 (2008).

${ }^{3}$ G. X. Ding, D. M. Duggan, and C. W. Coffey, "A theoretical approach for non-equilibrium radiation dosimetry," Phys. Med. Biol. 53, 3493-3499 (2008).

${ }^{4}$ E. Pappas, T. G. Maris, A. Papadakis, F. Zacharopoulou, J. Damilakis, N. Papanikolaou, and N. Gourtsoyiannis, "Experimental determination of the effect of detector size on profile measurements in narrow photon beams," Med. Phys. 33, 3700-3710 (2006).

${ }^{5}$ M. Bucciolini, F. B. Buonamici, S. Mazzocchi, C. De Angelis, S. Onori, and G. A. P. Cirrone, "Diamond detector versus silicon diode and ion chamber in photon beams of different energy and field size," Med. Phys. 30, 2149-2154 (2003).

${ }^{6}$ S. Almaviva, I. Ciancaglioni, R. Consorti, F. De Notaristefani, C. Manfredotti, M. Marinelli, E. Milani, A. Petrucci, G. Prestopino, C. Verona, and G. Verona-Rinati, "Synthetic single crystal diamond dosimeter for intensity modulated radiation therapy applications," Nucl. Instrum. Methods A 608, 191-194 (2009).

${ }^{7}$ R. P. D. Tromson et al., "Single crystal CVD diamond detector for high resolution dose measurement for IMRT and novel radiation therapy needs," Diamond Relat. Mater. 19, 1012-1021 (2010).

${ }^{8}$ M. Bruzzi, C. De Angelis, M. Scaringella, C. Talamonti, D. Viscomi, and M. Bucciolini, "Zero-bias operation of polycrystalline chemically vapour deposited diamond films for intensity modulated radiation therapy," Diamond Relat. Mater. 20, 84-92 (2011).

${ }^{9}$ I. Ciancaglioni, M. Marinelli, E. Milani, G. Prestopino, C. Verona, G. Verona-Rinati, R. Consorti, A. Petrucci, and F. D. Notaristefani, "Dosimetric characterization of a synthetic single crystal diamond detector in clinical radiation therapy small photon beams," Med. Phys. 39, 4493-4501 (2012).

${ }^{10}$ E. Barnett, M. MacKenzie, and B. G. Fallone, "IMRT point dose measurements with a diamond detector," Radial. Oncol. 39, 71-78 (2005).

${ }^{11}$ D. A. Low, J. M. Moran, J. F. Dempsey, L. Dong, and M. Oldham, "Dosimetry tools and techniques for IMRT," Med. Phys. 38, 1313-1338 (2011).

${ }^{12}$ C. De Angelis, M. Casati, M. Bruzzi, S. Onori, and M. Bucciolini, "Present limitations of CVD diamond detectors for IMRT applications," Nucl. Instrum. Methods Phys. Res. A 583, 195-203 (2007). 
${ }^{13}$ M. Bruzzi, C. Talamonti, M. Scaringella, E. Pace, A. D. Sio, L. Tozzetti, M. Zani, and M. Bucciolini, "IMRT field profiling by high-quality polycrystalline CVD diamond," JINST 7, P08003 (2012).

${ }^{14}$ C. De Angelis, M. Bucciolini, D. Viscomi, B. Marczewska, and S. Onori, "Characterization of a HPHT diamond detector for clinical applications," Nucl. Instrum. Methods Phys. Res. A 612, 576-579 (2010).

${ }^{15}$ S. Almaviva, E. Milani, A. Tucciarone, G. Verona-Rinati, A. Petrucci, F. De Notaristefani, and I. Ciancaglioni, "Synthetic single crystal diamond diodes for radiotherapy dosimetry," Nucl. Instrum. Methods Phys. Res. A 594, 273-277 (2008)

${ }^{16}$ M. Pimpinella, I. Ciancaglioni, R. Consorti, C. De Venanzio, A. S. Guerra, A. Petrucci, A. Stravato, and G. Verona-Rinati, "A synthetic diamond detector as transfer dosimeter for $D_{w}$ measurements in photon beams with small field sizes," Metrologia 49, s207-s210 (2012).

${ }^{17}$ C. De Venanzio, M. Marinelli, E. Milani, G. Prestopino, C. Verona, G. Verona-Rinati, M. D. Falco, P. Bagala, R. Santoni, and M. Pimpinella, "Characterization of a synthetic single crystal diamond Schottky diode for radiotherapy electron beam dosimetry," Med. Phys. 40, 021712 (9pp.) (2013).

${ }^{18}$ S. Amerio, A. Boriano, F. Bourhaleb, R. Cirio, M. Donetti, A. Fidanzio, E. Garelli, S. Giordanengo, E. Madon, F. Marchetto, U. Nastasi, C. Peroni, A. Piermattei, C. J. S. Freire, A. Sardo, and E. Trevisiol, "Dosimetric characterization of a large area pixel-segmented ionization chamber," Med. Phys. 31, 414-420 (2004).
${ }^{19}$ M. Stasi, S. Giordanengo, R. Cirio, A. Boriano, F. Bourhaleb, I. Cornelius, M. Donetti, E. Garelli, I. Gomola, F. Marchetto, M. Porzio, C. S. Freire, A. Sardo, and C. Peroni, "D-IMRT verification with a 2D pixel ionization chamber: Dosimetric and clinical results in head and neck cancer," Phys. Med. Biol. 50, 4681-4694 (2005).

${ }^{20}$ F. B. Buonamici, A. Compagnucci, L. Marrazzo, S. Russo, and M. Bucciolini, "An intercomparison between film dosimetry and diode matrix for IMRT quality assurance," Med. Phys. 34, 1372-1379 (2007).

${ }^{21}$ P. A. Jursinic and B. E. Nelm, "A 2-D diode array and analysis software for verification of intensity modulated radiation therapy deliver," Med. Phys. 30, 870-879 (2003).

${ }^{22}$ B. Poppe, A. Blechschmidt, A. Djouguela, R. Kollhoff, A. Rubach, K. C. Willborn, and D. Harder, "Two-dimensional ionization chamber arrays for IMRT plan verification," Med. Phys. 33, 1005-1015 (2006).

${ }^{23}$ IAEA, "Absorbed dose in external beam radiotherapy: An international code of practice for dosimetry based on standards aof absorbed dose to water," IAEA Technical Reports Series 398 (IAEA, Vienna, 2001).

${ }^{24}$ J. V. Dyk, R. B. Barnett, J. E. Cygler, and P. C. Shragge, "Commissioning and quality assurance of treatment planning computers," Int. J. Radiat. Oncol., Biol., Phys. 26, 261-273 (1993).

${ }^{25}$ W. B. Harms, S. I. Low, J. W. Wong, and J. A. Purdy, "A software tool for the evaluation of 3D dose calculation algorithms," Med. Phys. 25, 18301836 (1998). 